\title{
Cosmological Vorticity in a Gravity with Quadratic Order Curvature Couplings
}

\author{
Jai-chan Hwang \\ Department of Astronomy and Atmospheric Sciences, Kyungpook National University, Taegu, Korea \\ Hyerim Noh \\ Korea Astronomy Observatory, San 36-1, Whaam-dong, Yusung-gu, Daejon, Korea
}

(November 10, 2017)

\begin{abstract}
We analyse the evolution of the rotational type cosmological perturbation in a gravity with general quadratic order gravitational coupling terms. The result is expressed independently of the generalized nature of the gravity theory, and is simply interpreted as a conservation of the angular momentum.
\end{abstract}

PACS numbers: 04.50.+h, 04.62.+v, 98.80.-k, 98.80.Hw

\section{INTRODUCTION}

In a series of work we have been investigating the evolution of cosmological perturbations in a gravity with quadratic order curvature correction terms

$$
S=\int d^{4} x \sqrt{-g}\left[\frac{1}{2}\left(R+A R^{2}+B R^{a b} R_{a b}\right)+L_{m}\right]
$$

where $L_{m}$ is the matter part Lagrangian. The gravitational field equation is presented in Eq. (2) of [1]. In contrast with the situation with generalized $f(\phi, R)$ gravity in Eq. (27), the gravity with Ricci-curvature square term in the action does not have the conformal symmetry to Einstein gravity [2]. In [1] we analysed the gravitational wave and derived a fourth order differential equation. However, for the background equation we have a symmetry between the $R^{2}$ and $R^{a b} R_{a b}$ terms; the effects of the two terms appear in a common manner in the background equation, see Eq. 222.

In this paper, we will analyse the vector type perturbation. In contrast with the gravitational wave studied in [1] we will find that the Ricci-curvature square term does not add any new feature to the evolution of the vector type perturbation: the rotational perturbation is described by the angular momentum conservation independently of the generalized nature of the gravity theory. The main result is Eq. (26) which is the same equation valid in Einstein gravity with $A=0=B$.

Since the analysis for $R^{a b} R_{a b}$ term is new, we present the necessary details for the derivation in the Appendices. We take the same convention as [1].

\section{THE ROTATIONAL TYPE PERTURBATION}

As the background universe model we consider a spatially flat, homogeneous and isotropic spacetime. Out of the three (the scalar, vector and tensor) types of general perturbations, in this paper, we consider only the transverse vector type perturbation. To the linear order, due to the symmetry in the background, the three types of perturbations decouple from each other and evolve independently. The tensor type perturbation was considered in [1], and the scalar type of perturbation will be considered in future. The cosmological metric with the general vector type perturbation is

$$
\begin{aligned}
d s^{2}= & -a^{2} d \eta^{2}-a^{2} B_{\alpha} d \eta d x^{\alpha} \\
& +a^{2}\left(\delta_{\alpha \beta}+2 C_{(\alpha, \beta)}\right) d x^{\alpha} d x^{\beta},
\end{aligned}
$$

where $a(\eta)$ is a cosmic scale factor. $B_{\alpha}(\mathbf{x}, \eta)$ and $C_{\alpha}(\mathbf{x}, \eta)$ are transverse perturbed order variables based on a met$\operatorname{ric} \delta_{\alpha \beta}$. Except for the gauge redundancy, these variables represent the rotational type metric perturbations with $B^{\alpha}{ }_{, \alpha} \equiv 0 \equiv C^{\alpha}{ }_{, \alpha}$. The inverse metric, the connection, and the curvatures based on the metric in Eq. (2), which are valid to the linear order in $B_{\alpha}$ and $C_{\alpha}$, are summarized in the Appendices.

Now, we discuss the energy momentum contents supporting the rotational perturbation. The variation of the matter part Lagrangian leads to the energy momentum tensor as $\delta\left(\sqrt{-g} L_{m}\right) \equiv \frac{1}{2} \sqrt{-g} T^{a b} \delta g_{a b}$. We can decompose the energy momentum tensor covariantly as

$$
T_{a b}=\mu u_{a} u_{b}+p h_{a b}+q_{a} u_{b}+q_{b} u_{a}+\pi_{a b},
$$

where $h_{a b} \equiv g_{a b}+u_{a} u_{b}, q_{a} u^{a} \equiv 0 \equiv \pi_{a b} u^{b}$, and $\pi_{a}^{a} \equiv 0$; for the covariant set of equations, see [3]. The four vector $u_{a}$ is decomposed as

$$
\begin{aligned}
& u^{0} \equiv a^{-1}, \quad u_{0}=-a, \\
& u^{\alpha} \equiv a^{-1} V^{\alpha}, \quad u_{\alpha}=a\left(V_{\alpha}-B_{\alpha}\right) .
\end{aligned}
$$

We decompose the energy flux $q_{a}$ as $q_{0}=0$ and $q_{\alpha} \equiv$ $a Q_{\alpha} . V_{\alpha}$ and $Q_{\alpha}$ are based on $\delta_{\alpha \beta}$ with $V^{\alpha}{ }_{, \alpha} \equiv 0 \equiv Q^{\alpha}{ }_{, \alpha}$. In the perturbed set of equations, $V_{\alpha}$ and $Q_{\alpha}$ always appear together in a combination $V_{\alpha}+Q_{\alpha} /(\mu+p)$. The energy frame condition $q_{\alpha} \equiv 0$ corresponds to taking 
$Q_{\alpha} \equiv 0$, whereas the normal frame condition $u_{\alpha} \equiv 0$ corresponds to taking $V_{\alpha}-B_{\alpha} \equiv 0$, [4]. The combination $V_{\alpha}+Q_{\alpha} /(\mu+p)$ counts for the general dependence on frames. The vorticity tensor $\bar{\omega}_{a b}$ and the shear tensor $\bar{\sigma}_{a b}$ of the four vector flow $u_{a}$ are introduced as [3]

$$
\bar{\omega}_{a b} \equiv h_{[a}^{c} h_{b]}^{d} u_{c ; d}, \quad \bar{\sigma}_{a b} \equiv h_{(a}^{c} h_{b)}^{d} u_{c ; d}-\frac{1}{3} h_{a b} u_{; c}^{c},
$$

where $t_{[a b]} \equiv \frac{1}{2}\left(t_{a b}-t_{b a}\right)$ and $t_{(a b)} \equiv \frac{1}{2}\left(t_{a b}+t_{b a}\right)$. The vorticity tensor $\omega_{a b}$ and the shear tensor $\sigma_{a b}$ based on the frame invariant four vector flow $u_{\alpha}^{E} \equiv u_{\alpha}+q_{\alpha} /(\mu+p)$ can be shown as

$$
\begin{aligned}
& \omega_{\alpha \beta}=u_{[\alpha, \beta]}^{E}=a\left(V_{[\alpha, \beta]}+\frac{Q_{[\alpha, \beta]}}{\mu+p}-B_{[\alpha, \beta]}\right), \\
& \sigma_{\alpha \beta}=a\left(V_{(\alpha, \beta)}+\frac{Q_{(\alpha, \beta)}}{\mu+p}+C_{(\alpha, \beta)}^{\prime}\right) .
\end{aligned}
$$

The energy momentum tensor becomes

$$
T_{\alpha}^{0}=(\mu+p)\left(V_{\alpha}+\frac{Q_{\alpha}}{\mu+p}-B_{\alpha}\right), \quad \delta T_{\beta}^{\alpha}=\Pi_{\beta}^{\alpha},
$$

where we let $\pi_{\beta}^{\alpha} \equiv \Pi_{\beta}^{\alpha}$ and consider $\Pi_{\beta}^{\alpha}$ as based on $\delta_{\alpha \beta}$. $\mu$ and $p$ are the energy density and the pressure based on $u_{a}$ as

$$
\mu \equiv T_{a b} u^{a} u^{b}=-T_{0}^{0}, \quad p \equiv \frac{1}{3} T_{a b} h^{a b}=\frac{1}{3} T_{\alpha}^{\alpha} .
$$

To the linear order, $\mu$ and $p$ are frame invariant, [4].

\section{THE GAUGE ISSUE}

Under the gauge transformation $\hat{x}^{a}=x^{a}+\tilde{\xi}^{a}\left(x^{e}\right)$ the metric transforms as

$$
\delta \hat{g}_{a b}=\delta g_{a b}-g_{a b, c} \tilde{\xi}^{c}-g_{c b} \tilde{\xi}_{, a}^{c}-g_{a c} \tilde{\xi}^{c}{ }_{, b},
$$

and similarly for the energy momentum tensor $T_{a b}$. We consider the vector type transformation, thus $\tilde{\xi}^{0} \equiv 0$ and $\tilde{\xi}^{\alpha} \equiv \xi^{\alpha}$ with $\xi^{\alpha}$ based on $\delta_{\alpha \beta}$ and $\xi^{\alpha}{ }_{, \alpha} \equiv 0$. From the gauge transformation properties of the metric and the energy momentum tensor we can show that

$$
\begin{aligned}
& \hat{B}_{\alpha}=B_{\alpha}+\xi_{\alpha}^{\prime}, \quad \hat{C}_{\alpha}=C_{\alpha}-\xi_{\alpha}, \\
& \hat{V}_{\alpha}+\frac{\hat{Q}_{\alpha}}{\mu+p}=V_{\alpha}+\frac{Q_{\alpha}}{\mu+p}+\xi_{\alpha}^{\prime},
\end{aligned}
$$

and $\Pi_{\beta}^{\alpha}$ is gauge invariant. Thus, we have three different gauge conditions: the condition $B_{\alpha} \equiv 0$ or $V_{\alpha}+Q_{\alpha} /(\mu+p) \equiv 0$ does not fix the gauge condition completely, whereas, the $C_{\alpha} \equiv 0$ condition completely fixes the gauge condition. Thus, without losing generality we can take $C_{\alpha} \equiv 0$ as a preferred gauge condition; we call this the $\mathrm{C}$-gauge condition, [5]. Under the $\mathrm{C}$-gauge condition, $B_{\alpha}$ and $V_{\alpha}+Q_{\alpha} /(\mu+p)$ are the same as the following gauge invariant combinations, respectively

$$
B_{\alpha}+C_{\alpha}^{\prime}, \quad V_{\alpha}+\frac{Q_{\alpha}}{\mu+p}+C_{\alpha}^{\prime}
$$

We have one more gauge invariant combination

$$
V_{\alpha}+\frac{Q_{\alpha}}{\mu+p}-B_{\alpha}
$$

\section{HARMONIC DECOMPOSITION}

We treat the spatial dependence of the perturbed variables using the vector type harmonic function $Y_{\alpha}$ introduced as 6

$$
\Delta Y_{\alpha} \equiv-k^{2} Y_{\alpha}, \quad Y_{\alpha \beta} \equiv-\frac{1}{k} Y_{(\alpha, \beta)}, \quad Y_{, \alpha}^{\alpha} \equiv 0 .
$$

We decompose the perturbation variables as

$$
\begin{aligned}
& B_{\alpha}(\mathbf{x}, t) \equiv b(t) Y_{\alpha}(\mathbf{x}), \quad C_{\alpha} \equiv c Y_{\alpha} \\
& V_{\alpha} \equiv v Y_{\alpha}, \quad Q_{\alpha} \equiv q Y_{\alpha}, \quad \Pi_{\beta}^{\alpha} \equiv p \pi_{T} Y_{\beta}^{\alpha}
\end{aligned}
$$

We introduce the following gauge invariant variables:

$$
\begin{aligned}
& V_{\alpha}+\frac{Q_{\alpha}}{\mu+p}-B_{\alpha}=\left(v+\frac{q}{\mu+p}-b\right) Y_{\alpha} \equiv v_{\omega} Y_{\alpha} \\
& V_{\alpha}+\frac{Q_{\alpha}}{\mu+p}+C_{\alpha}^{\prime}=\left(v+\frac{q}{\mu+p}+c^{\prime}\right) Y_{\alpha} \equiv v_{\sigma} Y_{\alpha} \\
& B_{\alpha}+C_{\alpha}^{\prime}=\left(v_{\sigma}-v_{\omega}\right) Y_{\alpha} \equiv \Psi Y_{\alpha} .
\end{aligned}
$$

From Eq. (16) we have

$$
\omega_{\alpha \beta}=a v_{\omega} Y_{[\alpha, \beta]}, \quad \sigma_{\alpha \beta}=a v_{\sigma} Y_{(\alpha, \beta)} .
$$

The amplitude square of the vorticity and the shear are defined as

$$
\omega^{2} \equiv \frac{1}{2} \omega^{a b} \omega_{a b}, \quad \sigma^{2} \equiv \frac{1}{2} \sigma^{a b} \sigma_{a b}
$$

We can show

$$
\begin{aligned}
\omega & =\left|\frac{1}{a} v_{\omega}\right| \sqrt{\frac{1}{2} Y^{\alpha \mid \beta} Y_{[\alpha, \beta]}}, \\
\sigma & =\left|\frac{1}{a} v_{\sigma}\right| \sqrt{\frac{1}{2} Y^{\alpha \mid \beta} Y_{(\alpha, \beta)}} .
\end{aligned}
$$

Thus,

$$
v_{\omega} \propto a \omega, \quad v_{\sigma} \propto a \sigma,
$$

and we can interprete $v_{\omega}$ and $v_{\sigma}$ as the velocity variables related to the vorticity and the shear, respectively; in [6,2,7] $v_{\omega}$ and $v_{\sigma}$ are written as $v_{c}$ and $v_{s}$, respectively. 


\section{EQUATIONS FOR THE COSMOLOGICAL VORTICITY}

Using the quantities presented in the Appendix B (thus taking the C-gauge) and Eq. (7), the gravitational field equation in Eq. (2) of [1] becomes:

$$
\begin{aligned}
\delta T_{\alpha}^{0}= & (1+2 A R) R_{\alpha}^{0} \\
& -B\left[\ddot{R}_{\alpha}^{0}+5 H \dot{R}_{\alpha}^{0}-\left(2 \dot{H}+2 H^{2}+\frac{\Delta}{a^{2}}\right) R_{\alpha}^{0}\right] \\
= & (\mu+p)\left(V_{\alpha}+\frac{Q_{\alpha}}{\mu+p}-B_{\alpha}\right), \\
\delta T_{\beta}^{\alpha}= & \frac{1}{2 a^{3}}\left[(1+2 A R) a^{2}\left(B_{, \beta}^{\alpha}+B_{\beta}{ }^{\mid \alpha}\right)\right] \\
& -B\left[\delta \ddot{R}_{\beta}^{\alpha}+3 H \delta \dot{R}_{\beta}^{\alpha}-\left(6 H^{2}+6 \dot{H}+\frac{\Delta}{a^{2}}\right) \delta R_{\beta}^{\alpha}\right. \\
& \left.+\left(-6 H \dot{H}-3 \ddot{H}+H \frac{\Delta}{a^{2}}\right) \frac{1}{a}\left(B_{, \beta}^{\alpha}+B_{\beta}{ }^{\mid \alpha}\right)\right] \\
= & \Pi_{\beta}^{\alpha},
\end{aligned}
$$

where $R=6\left(\dot{H}+2 H^{2}\right)$. The equations for the background are derived in Eq. (7) of [1]:

$$
\begin{aligned}
& H^{2}+2(3 A+B)\left(2 H \ddot{H}-\dot{H}^{2}+6 H^{2} \dot{H}\right)=\frac{1}{3} \mu, \\
& \dot{\mu}=-3 H(\mu+p)
\end{aligned}
$$

From Eqs. $36,15,13,17,14$ we have

$$
\begin{aligned}
& R_{\alpha}^{0}=\frac{k^{2}}{2 a^{2}} \Psi Y_{\alpha}, \quad \delta R_{\beta}^{\alpha}=-\frac{k}{a^{3}}\left(a^{2} \Psi\right)^{\cdot} Y_{\beta}^{\alpha}, \\
& T_{\alpha}^{0}=(\mu+p) v_{\omega} Y_{\alpha}, \quad \delta T_{\beta}^{\alpha}=p \pi_{T} Y_{\beta}^{\alpha}
\end{aligned}
$$

and Eqs. 20,21) can be combined to give

$$
\begin{aligned}
& \frac{k^{2}}{2 a^{2}}\left\{(1+2 A R) \Psi-B\left[\ddot{\Psi}+H \dot{\Psi}-\left(\frac{2}{3} R+\frac{\Delta}{a^{2}}\right) \Psi\right]\right\} \\
& \quad=(\mu+p) v_{\omega}, \\
& \frac{1}{a^{4}}\left[a^{4}(\mu+p) v_{\omega}\right]=-\frac{k}{2 a} p \pi_{T} .
\end{aligned}
$$

Equation (26) implies the angular momentum conservation: for $p \pi_{T}=0$ we have 'angular momentum' $\sim a^{3}(\mu+p) \times a \times v_{\omega} \sim$ constant in time. Notice that, remarkably, the angular momentum conservation relation in Eq. (26) involves neither $A$ nor $B$ in Eq. (11), thus, does not depend on the character of the generalized nature of the gravity theories.

\section{DISCUSSIONS}

The cosmological evolution of the rotational perturbation in Einstein gravity and its simple characterization by the angular momentum conservation were first presented in a classic study by Lifshitz in 8. The extension of the result to the generalized gravity with an action

$$
\begin{gathered}
S=\int d^{4} x \sqrt{-g}\left[\frac{1}{2} f(\phi, R)-\frac{1}{2} \omega(\phi) \phi^{; a} \phi_{, a}-V(\phi)\right. \\
\left.+L_{m}\right],
\end{gathered}
$$

was presented in [2]7]; $\phi$ is a dilaton field. In this case, instead of Eq. (25), we have

$$
\frac{k^{2}}{2 a^{2}} F \Psi=(\mu+p) v_{\omega},
$$

where $F \equiv \partial f /(\partial R)$, and Eq. (26) remains intact. For the gravity in Eq. (11) with $B=0$, we have $F=1+2 A R$. In [9] 10 the authors considered instabilities of the homogeneous and isotropic world model based on $L \sim f(R)$ gravity under the gravitational wave and rotational perturbations. The results of both the gravitational wave and rotational perturbations agree with [2,1] and present work, respectively. In [9] the author analysed the scalar type perturbations in $L \sim R^{n}$ gravity using the synchronous gauge; recent progress in 111 successfully resolved the issue in a simple and unified manner in the gravity theories in Eq. (27) using the uniform-curvature gauge. [The referee has informed us the important works in 9.10.$]$

The equation for a multi-component situation in $L_{m}$ in the context of Eq. (27) is presented in Sec. 5.1 of [7]. The evolution of background universe follows Eq. (22) where the fluid quantities, $\mu$ and $p$, concern the matter part Lagrangian. For $p \equiv \mathrm{w} \mu$ with $\mathrm{w}=$ constant we have $\mu \propto a^{-3(1+\mathrm{w})}$. Thus, for vanishing anisotropic pressure of the matter part, $p \pi_{T}=0$, we have

$$
v_{\omega} \propto a^{-1+3 \mathrm{w}},
$$

thus, $v_{\omega} \propto a^{0}, a^{-1}, a^{-4}$ for the radiation flow ( $\mathrm{w}=\frac{1}{3}$ ), the matter flow $(\mathrm{w}=0)$, and the inflationary matter flow $(\mathrm{w} \simeq-1)$, respectively. This result concerning the vorticity does not depend on the generalized nature of the gravity theories of the types in Eqs. (1, 27). The related disturbances in the metric $(\Psi)$ and the corresponding shear of the flow $\left(v_{\sigma}\right)$ do, however, depend on the generalized nature of the gravity as in Eqs. (25,28).

\section{ACKNOWLEDGMENTS}

This work was supported by the KOSEF, Grant No. 95-0702-04-01-3 and through the SRC program of SNUCTP.

APPENDIX A: LINEAR ORDER QUANTITIES 
The inverse metric $(0=\eta)$ :

$$
\begin{aligned}
& g^{00}=-\frac{1}{a^{2}}, \quad g^{0 \alpha}=-\frac{1}{a^{2}} B^{\alpha}, \\
& g^{\alpha \beta}=\frac{1}{a^{2}}\left(\delta^{\alpha \beta}-2 C^{(\alpha \mid \beta)}\right) .
\end{aligned}
$$

The connection:

$$
\begin{aligned}
& \Gamma_{00}^{0}=\frac{a^{\prime}}{a}, \quad \Gamma_{0 \alpha}^{0}=-\frac{a^{\prime}}{a} B_{\alpha}, \quad \Gamma_{00}^{\alpha}=-B^{\alpha \prime}-\frac{a^{\prime}}{a} B^{\alpha} \\
& \Gamma_{\alpha \beta}^{0}=\frac{a^{\prime}}{a} \delta_{\alpha \beta}+B_{(\alpha, \beta)}+C_{(\alpha, \beta)}^{\prime}+2 \frac{a^{\prime}}{a} C_{(\alpha, \beta)} \\
& \Gamma_{0 \beta}^{\alpha}=\frac{a^{\prime}}{a} \delta_{\beta}^{\alpha}-\frac{1}{2}\left(B_{, \beta}^{\alpha}-B_{\beta}^{\mid \alpha}\right)+\frac{1}{2}\left(C_{, \beta}^{\alpha}+C_{\beta}^{\mid \alpha}\right)^{\prime} \\
& \Gamma_{\beta \gamma}^{\alpha}=\frac{a^{\prime}}{a} B^{\alpha} \delta_{\beta \gamma}+C_{, \beta \gamma}^{\alpha} .
\end{aligned}
$$

Riemann curvature:

$$
\begin{aligned}
& R_{b 00}^{a}=0=R_{0 \alpha \beta}^{0}, \quad R_{00 \alpha}^{0}=-\left(\frac{a^{\prime}}{a}\right)^{\prime} B_{\alpha}, \\
& R_{\alpha 0 \beta}^{0}=\left(\frac{a^{\prime}}{a}\right)^{\prime} \delta_{\alpha \beta}+B_{(\alpha, \beta)}^{\prime}+\frac{a^{\prime}}{a} B_{(\alpha, \beta)} \\
& +C_{(\alpha, \beta)}^{\prime \prime}+\frac{a^{\prime}}{a} C_{(\alpha, \beta)}^{\prime}+2\left(\frac{a^{\prime}}{a}\right)^{\prime} C_{(\alpha, \beta)}, \\
& R_{\alpha \beta \gamma}^{0}=B_{[\gamma, \beta] \alpha}+C_{[\gamma, \beta] \alpha}^{\prime}, \\
& R_{00 \beta}^{\alpha}=\left(\frac{a^{\prime}}{a}\right)^{\prime} \delta_{\beta}^{\alpha} \\
& +\frac{1}{2}\left(B_{, \beta}^{\alpha}+B_{\beta}^{\mid \alpha}\right)^{\prime}+\frac{1}{2} \frac{a^{\prime}}{a}\left(B_{, \beta}^{\alpha}+B_{\beta}^{\mid \alpha}\right) \\
& +\frac{1}{2}\left(C_{, \beta}^{\alpha}+C_{\beta}^{\mid \alpha}\right)^{\prime \prime}+\frac{1}{2} \frac{a^{\prime}}{a}\left(C_{, \beta}^{\alpha}+C_{\beta}^{\mid \alpha}\right)^{\prime} \text {, } \\
& R^{\alpha \beta \gamma}=B_{[\gamma, \beta]}^{\mid \alpha}+2\left(\frac{a^{\prime}}{a}\right)^{2} \delta_{[\gamma}^{\alpha} B_{\beta]}+C_{[\gamma, \beta]}^{\prime \mid \alpha}, \\
& R_{\beta 0 \gamma}^{\alpha}=\left(\frac{a^{\prime}}{a}\right)^{\prime} B^{\alpha} \delta_{\beta \gamma}+\left(\frac{a^{\prime}}{a}\right)^{2}\left(-B^{\alpha} \delta_{\beta \gamma}+B_{\beta} \delta_{\gamma}^{\alpha}\right) \\
& +\frac{1}{2}\left(B_{, \beta}^{\alpha}-B_{\beta}^{\mid \alpha}\right)_{, \gamma}+\frac{1}{2}\left(C_{, \beta}^{\alpha}-C_{\beta}^{\mid \alpha}\right)_{, \gamma}^{\prime}, \\
& R_{\beta \gamma \delta}^{\alpha}=2\left(\frac{a^{\prime}}{a}\right)^{2} \delta_{[\gamma}^{\alpha} \delta_{\delta] \beta} \\
& +\frac{a^{\prime}}{a}\left(\delta_{[\gamma}^{\alpha} B_{\delta], \beta}+B_{\beta,[\delta} \delta_{\gamma]}^{\alpha}+\delta_{[\gamma}^{\alpha} C_{\delta], \beta}^{\prime}+C_{\beta,[\delta}^{\prime} \delta_{\gamma]}^{\alpha}\right) \\
& +2\left(\frac{a^{\prime}}{a}\right)^{2}\left(\delta_{[\gamma}^{\alpha} C_{\delta], \beta}+C_{\beta,[\delta} \delta_{\gamma]}^{\alpha}\right) \\
& +\frac{a^{\prime}}{a} \delta_{\beta[\delta}\left[B_{, \gamma]}^{\alpha}+B_{\gamma]}{ }^{\mid \alpha}+\left(C_{, \gamma]}^{\alpha}+C_{\gamma]}{ }^{\mid \alpha}\right)^{\prime}\right] \text {. }
\end{aligned}
$$

In the following and the Appendix B we present only the perturbed parts and ignore the $(0,0)$ components which give null results to the perturbed order; the results valid in the background order are presented in the Appendices of [1].
Ricci curvature: $\delta R_{0}^{0}=0$,

$$
\begin{aligned}
R_{\alpha}^{0}= & -\frac{1}{2} \frac{\Delta}{a^{2}}\left(B_{\alpha}+C_{\alpha}^{\prime}\right), \\
\delta R_{\beta}^{\alpha} & =\frac{1}{a^{2}}\left\{\frac{1}{2}\left(B_{, \beta}^{\alpha}+B_{\beta}^{\mid \alpha}\right)^{\prime}+\frac{a^{\prime}}{a}\left(B_{, \beta}^{\alpha}+B_{\beta}^{\mid \alpha}\right)\right. \\
& \left.+\frac{1}{2}\left(C_{, \beta}^{\alpha}+C_{\beta}^{\mid \alpha}\right)^{\prime \prime}+\frac{a^{\prime}}{a}\left(C_{, \beta}^{\alpha}+C_{\beta}^{\mid \alpha}\right)^{\prime}\right\} .
\end{aligned}
$$

Scalar curvature: $\delta R=0$.

Curvature combinations:

$$
\begin{aligned}
& \delta\left(R_{b}^{a} R_{a}^{b}\right)=0=\delta\left(R^{c d} R_{c d 0}^{0}\right), \\
& R^{c d} R_{c d \alpha}^{0}=\left(\frac{a^{\prime}}{a}\right)^{2} \frac{\Delta}{a^{4}}\left(B_{\alpha}+C_{\alpha}^{\prime}\right), \\
& \delta\left(R^{c d} R_{c d \beta}^{\alpha}\right)=-\frac{2}{a^{4}} \frac{a^{\prime}}{a}\left(\frac{a^{\prime}}{a}\right)^{\prime} \\
& \times\left[B_{, \beta}^{\alpha}+B_{\beta}^{\mid \alpha}+\left(C_{, \beta}^{\alpha}+C_{\beta}^{\mid \alpha}\right)^{\prime}\right] \\
& +\frac{1}{2 a^{4}}\left[-3\left(\frac{a^{\prime}}{a}\right)^{\prime}+\left(\frac{a^{\prime}}{a}\right)^{2}\right] \\
& \times\left[\left(B_{, \beta}^{\alpha}+B_{\beta}^{\mid \alpha}\right)^{\prime}+\left(C_{, \beta}^{\alpha}+C_{\beta}^{\mid \alpha}\right)^{\prime \prime}\right] .
\end{aligned}
$$

Covariant derivatives:

$$
\begin{aligned}
& \delta\left(R_{0}^{; 0}\right)=0=R_{\alpha}^{; 0}, \\
& \delta\left(R_{\beta}^{; \alpha}\right)=-\frac{1}{2 a^{2}} R^{\prime}\left[B_{, \beta}^{\alpha}+B_{\beta}^{\mid \alpha}+\left(C_{, \beta}^{\alpha}+C_{\beta}^{\mid \alpha}\right)^{\prime}\right], \\
& \square R=0=\square R_{0}^{0}, \\
& \square R_{\alpha}^{0}=\frac{1}{a^{2}}\left\{-R_{\alpha}^{0 \prime \prime}-4 \frac{a^{\prime}}{a} R_{\alpha}^{0 \prime}\right. \\
& \left.+\left[\Delta+2\left(\frac{a^{\prime}}{a}\right)^{\prime}-4\left(\frac{a^{\prime}}{a}\right)^{2}\right] R_{\alpha}^{0}\right\}, \\
& \delta\left(\square R_{\beta}^{\alpha}\right) \\
& =\frac{1}{a^{2}}\left\{-\delta R_{\beta}^{\alpha \prime \prime}-2 \frac{a^{\prime}}{a} \delta R_{\beta}^{\alpha \prime}+\left[\Delta+2\left(\frac{a^{\prime}}{a}\right)^{2}\right] \delta R_{\beta}^{\alpha}\right. \\
& \quad-\frac{a^{\prime}}{a^{3}}\left[\Delta+4\left(\frac{a^{\prime}}{a}\right)^{\prime}-4\left(\frac{a^{\prime}}{a}\right)^{2}\right] \\
& \times\left[B_{, \beta}^{\alpha}+B_{\beta}^{\mid \alpha}+\left(C_{, \beta}^{\alpha}+C_{\beta}{ }^{\alpha}{ }^{\alpha}{ }^{\prime}\right]\right\} .
\end{aligned}
$$

\section{APPENDIX B: IN TERMS OF $t$}

Using $t$ as the time variable $(d t=a d \eta)$, and taking $C_{\alpha} \equiv 0$ as the gauge condition, we have $(H \equiv \dot{a} / a)$ : 


$$
\begin{aligned}
& R_{\alpha}^{0}=-\frac{1}{2} \frac{\Delta}{a^{2}} B_{\alpha}, \quad \delta R_{\beta}^{\alpha}=\frac{1}{2 a^{3}}\left[a^{2}\left(B_{, \beta}^{\alpha}+B_{\beta}{ }^{\alpha}\right)\right], \\
& \delta R=0, \quad \delta\left(R_{b}^{a} R_{a}^{b}\right)=0, \\
& R^{c d} R^{0}{ }_{c d \alpha}=H^{2} \frac{\Delta}{a^{2}} B_{\alpha}, \\
& \delta\left(R^{c d} R^{\alpha}{ }_{c d \beta}\right)=-\frac{1}{2}\left(3 \dot{H}+2 H^{2}\right)\left[\frac{1}{a}\left(B_{, \beta}^{\alpha}+B_{\beta}^{\mid \alpha}\right)\right] \\
& \quad-\frac{1}{2}\left(7 \dot{H}+6 H^{2}\right) H \frac{1}{a}\left(B_{, \beta}^{\alpha}+B_{\beta}^{\mid \alpha}\right), \\
& R_{\alpha}^{; 0}=0, \quad \delta\left(R^{; \alpha}{ }_{\beta}\right)=-\dot{R} \frac{1}{2 a}\left(B_{, \beta}^{\alpha}+B_{\beta}^{\mid \alpha}\right), \\
& \delta(\square R)=0, \\
& \square R_{\alpha}^{0}=-\ddot{R}_{\alpha}^{0}-5 H \dot{R}_{\alpha}^{0}+\left(2 \dot{H}-2 H^{2}+\frac{\Delta}{a^{2}}\right) R_{\alpha}^{0}, \\
& \delta\left(\square R_{\beta}^{\alpha}\right)=-\delta \ddot{R}_{\beta}^{\alpha}-3 H \delta \dot{R}_{\beta}^{\alpha}+\left(2 H^{2}+\frac{\Delta}{a^{2}}\right) \delta R_{\beta}^{\alpha} \\
& \quad-H\left(4 \dot{H}+\frac{\Delta}{a^{2}}\right) \frac{1}{a}\left(B_{, \beta}^{\alpha}+B_{\beta}^{\mid \alpha}\right) .
\end{aligned}
$$

[1] H. Noh and J. Hwang, Phys. Rev. D 55, 5222 (1997).

[2] J. Hwang, Class. Quantum Grav. 7, 1613 (1990).

[3] J. Ehlers, Gen. Rel. Grav. 25, 1225 (1993); G. F. R. Ellis in General Relativity and Cosmology, ed. R. K. Sachs (New York: Academic), p. 104 (1971); in Cargese Lectures in Physics, ed. E. Schatzmann (New York: Gordon and Breach), p. 1 (1973); The appendix of J. Hwang and E. T. Vishniac Astrophys. J. 353, 1 (1990).

[4] W. Israel, Ann. Phys. 100, 310 (1976).

[5] H. Noh and J. Hwang, Phys. Rev. D 52, 1970 (1995).

[6] J. M. Bardeen, Phys. Rev. D 22, 1882 (1980).

[7] J. Hwang, Astrophys. J. 375, 443 (1991).

[8] E. M. Lifshitz, J. Phys. (USSR) 10, 116 (1946); For a summary, see Sec. 116 of L. D. Landau and E. M. Lifshitz The Classical Theory of Fields, Fourth revised English edition (Pergamon press: Oxford) (1975).

[9] H. Nariai, Prog. Theor. Phys. 49, 165 (1973).

[10] H. Nariai and K. Tanabe, Prog. Theor. Phys. 50, 1099 (1973); H. Nariai, Prog. Theor. Phys. 51, 613 (1974).

[11] J. Hwang and H. Noh, Phys. Rev. D 54, 1460 (1996). 